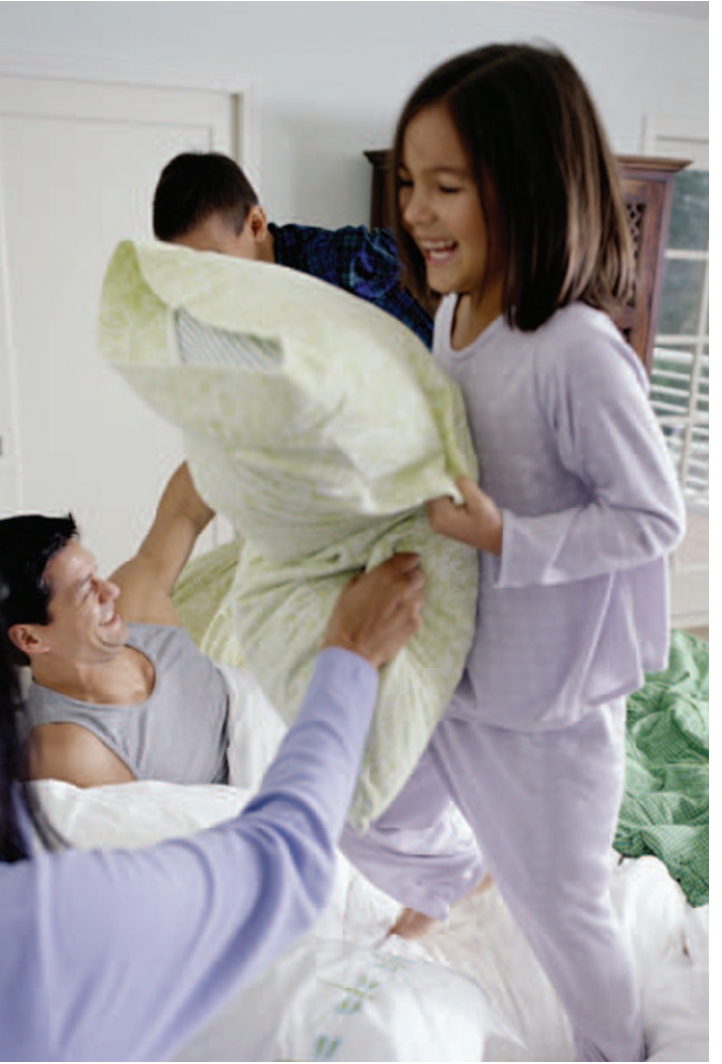

\section{Excess energy}

Upregulation of glucose-dependent metabolism, called aerobic glycolysis, is a feature of many tumours, especially those that are aggressive or at an advanced stage. Glycolysis provides the ATP to supply the tumour cell's high bioenergetic needs and, if there are excess metabolites, also provides the precursors for the synthesis of lipids that are required for membrane production and post-translational modification of proteins. In Cancer Cell, Craig Thompson and colleagues report that inhibition of a key enzyme linking glucose metabolism to lipid synthesis suppresses tumour growth.

Aerobic glycolysis produces pyruvate, which enters a tricarboxylic acid cycle in the mitochondria to produce citrate, which is in turn exported to the cytosol. Citrate is cleaved by ATP citrate lyase (ACLY) to produce acetyl-CoA, which is the main building block for lipids in the cell. The authors therefore assessed the effects of $A C L Y$ inhibition by short interfering RNA (siRNA) knockdown on tumour cells. Treatment of the human lung adenocarcinoma cell line A549 with anti-ACLY siRNA led to reduced levels of ACLY and acetyl-CoA, and a reduction in glucose-dependent lipid synthesis. By 72hours post-treatment, cells had accumulated in the G1 phase of the cell cycle, resulting in a 30\% decrease in cell numbers. A549 clones with either stable siRNA knockdown of ACLY or with vector-only transfection were injected into opposite flanks of nude mice to compare tumorigenicity. ACLY knockdown cells formed smaller, more differentiated tumours than the controls, marked by glandular structures that secreted mucin. The increased differentiation was a surprising finding, so the authors confirmed this effect with a wellknown model of tumour cell differentiation, the human chronic myelogenous leukaemia cell line, K562; ACLY knockdown also caused erythroid differentiation in these cells.

Thompson and colleagues also examined the effect of an ACLY chemical inhibitor SB204990 on tumour cell growth. Having shown that SB204990 reduced lipid synthesis and caused G1 arrest in a highly glycolytic

\title{
INK4C and medulloblastoma
}

\author{
Medulloblastoma is the most common \\ malignant paediatric brain tumour, but \\ the causative mutations in most of these \\ tumours are unknown. Evidence from \\ Martine Roussel and colleagues now \\ implicates loss of the tumour suppressor \\ INK4C as a causative agent in a significant \\ number of medulloblastoma cases. \\ The formation of the cerebellum mainly \\ occurs after birth, and involves the \\ proliferation of a thin layer of granule \\ neuronal precursor cells (GNPs) in response \\ to the mitogen sonic hedgehog (SHH). \\ After the requisite amount of proliferation, \\ the GNPs then exit the cell cycle and \\ differentiate. Evidence indicates that \\ mutated GNPs give rise to medulloblastoma, \\ and common mutations include a persistent \\ expression of proliferative proteins such as \\ NMYC, cyclin D1 and cyclin D2. \\ INK4C (encoded by CDKN2C) is a \\ specific inhibitor of cyclin D-dependent \\ kinases, and its expression profile in the \\ developing cerebellum indicates that \\ it might be important for regulating
}

the exit of GNPs from the cell cycle. So, Roussel and colleagues investigated the expression of INK4C in several mouse models of medulloblastoma. Using in situ hybridization, they found that $C d k n 2 c$ mRNA is present in dividing GNPs, and that its expression is transiently maintained as these cells exit the cell cycle and differentiate.

Loss of p53 is a useful method of inducing medulloblastoma in mice, although only $10 \%$ of human medulloblastomas have p53 mutations. Analyses in double-knockout Trp53 and Cdkn $2 c$ mice showed that GNPs in these mice remain in the cell cycle at later developmental stages than their wild-type counterparts. In vitro proliferation assays also showed that loss of $C d k n 2 c$ or Trp53 increased the time that GNPs remained sensitive to the mitogenic effects of SHH. Moreover, the combined inactivation of both genes resulted in an additive proliferative advantage.

As disruption of the Shh pathway occurs in approximately $25 \%$ of medulloblastoma cases, the authors examined the effect of INK4C disruption in mice that lack 1 allele of the $\mathrm{SHH}$ receptor patched (Ptch). They found that $P t c h 1^{+-}$mice that lacked either one or both $C d k n 2 c$ alleles showed earlier disease onset than $\mathrm{Ptch}^{+/-}, \mathrm{Cdkn2 \textrm {c } ^ { + / + }}$ mice. There was no difference in the tumour incidence rate between $C d k n 2 c$ heterozygotes or homozygotes, and loss of the second allele was not selected for in heterozygous tumours. This indicates that INK4C might be haploinsufficient in suppressing medulloblastoma development.

Is loss of INK4C important for human medulloblastoma development? The authors found that although CDKN2C mRNA is still expressed, 14 out of 73 samples showed no protein expression. This implies that alterations in the post-transcriptional regulation of INK4C might be important in a significant number of human medulloblastomas.

Nicola McCarthy

(2) References and links ORIGINAL RESEARCH PAPER Uziel, T. et al. The tumour suppressors Ink $4 \mathrm{C}$ and p 53 collaborate independently with Patched to suppress medulloblastoma formation. Genes Dev. 31 Oct 2005 (doi:10.1101/gad341605) WEB SITES

Martine Roussel's lab: http://www.stjude.org/ faculty/0,2512,407_2030_4116,00.html 
normal cell line, they went on to test it on three human tumour cell lines - two with high rates of glycolysis (A549 and PC3) were sensitive to SB204990, whereas one with a low rate of glycolysis (SKOV3) was more resistant. When mice bearing xenografts using these cell lines were treated with SB204990, a cytostatic effect as well as differentiation into glandular mucin-expressing structures was evident in A549 and PC3 xenografts but not SKOV3 xenografts.

The failure of ACLY inhibition to suppress the growth of tumours that do not have high rates of glycolysis indicates that there are alternative lipogenic pathways that tumour cells can use. However, ACLY inhibitors - as well as statins, farnesyl transferase inhibitors and fatty acid synthesis inhibitors - might be useful in combination with other agents to treat advanced tumours, which often have high rates of glycolysis.

\section{Ezzie Hutchinson}

\section{(0) References and links}

ORIGINAL RESEARCH PAPER Hatzivassiliou, G. et al. ATP citrate lyase inhibition can suppress tumor cell growth. Cancer Cell 8, 311-321 (2005)

WEB SITE

Craig Thompson's Lab:

http://www.uphs.upenn.edu/abramson/thompsonLab.html

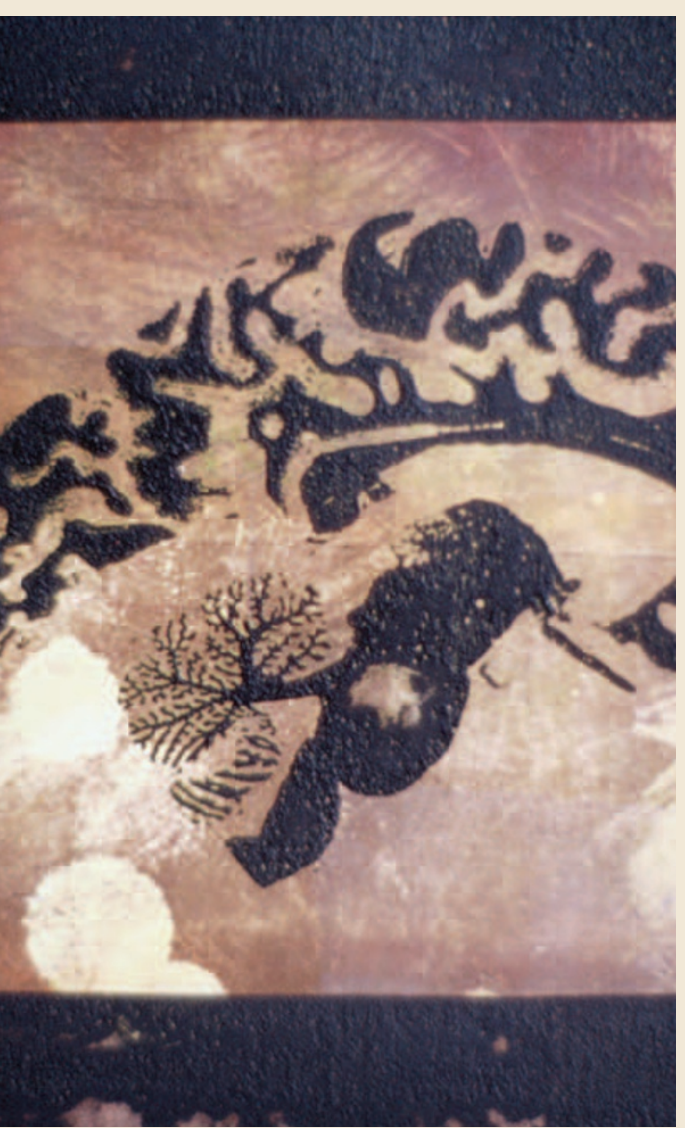

\section{TUMOUR SUPPRESSORS}

\section{Look both ways}

The Krüppel-like factor 4 (KLF4)

transcription factor can function as a tumour suppressor in various cancer types and as an oncogene in others, including breast cancer. In their Nature Cell Biology paper, Daniel Peeper and colleagues have identified some of the molecular mechanisms that explain the Janus-like behaviour of KLF4 in tumorigenesis.

When the authors identified KLF4 as a protein that can bypass cellular senescence induced by oncogenic RAS ( RAS $^{\mathrm{V} 12}$ ), they decided to investigate the molecular mechanisms that are at work. They established that KLF4 represses expression of the tumour suppressor p53, but induces expression of the cyclin-dependent-kinase inhibitor p21 (CDKN1A). p53 is a crucial mediator of $\mathrm{RAS}^{\mathrm{V} 12}$-induced proliferative arrest, so the authors hypothesized that suppression of $\mathrm{p} 53$ by KLF4 is probably the means through which KLF4 bypasses senescence. This was confirmed when short hairpin RNAs were used to suppress p53 to levels similar to those achieved by expression of KLF4, which prohibited RAS $^{\mathrm{V} 12}$-induced senescence.

How does KLF4 suppress the expression of p53? Northern blots demonstrated that KLF4 downregulates TP53 mRNA levels, and chromatin immunoprecipitation assays indicated that KLF4 achieves this by directly binding to the TP53 promoter.

Although KLF4-mediated repression of p53 explains how KLF4 prevents RAS ${ }^{\mathrm{V} 12}$ driven arrest, it does not explain the reverse: how RAS ${ }^{\mathrm{V} 12}$ bypasses KLF4-induced arrest. Indeed, KLF4, similar to most other tumour suppressors, normally triggers cell-cycle arrest. One of the known mitogenic targets of RAS is cyclin D1, and Peeper and colleagues found that RAS ${ }^{\mathrm{V} 12}$ could not collaborate with KLF4 in stimulating cell proliferation in the absence of cyclin D1 expression, indicating a crucial role for cyclin D1 in this setting.

How does this tie in with the expression of p21, which KLF4 induces? Cyclin D1 neutralizes the p21-induced cell-cycle arrest, so the cells are able to proliferate. In agreement with this hypothesis, p21null mouse embryonic fibroblasts were unable to arrest in the presence of KLF4. Together, these data indicate that KLF4 is a tumour suppressor that induces cell-

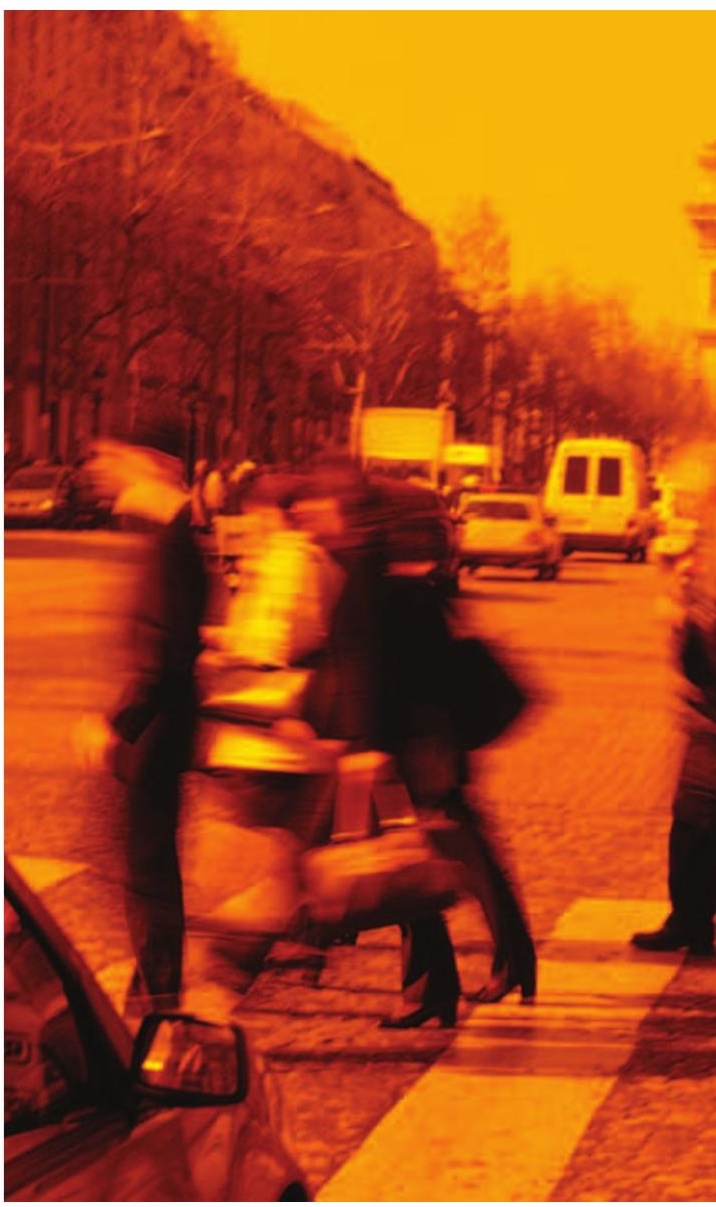

cycle arrest by increasing the expression level of p21.

The authors also examined whether the KLF4-mediated repression of p53 could explain the oncogenic behaviour of KLF4 in breast cancer cells. They found that suppressing KLF4 in human breast cancer cell lines restored the expression of $\mathrm{p} 53$, which resulted in apoptosis. So, suppression of p53 by KLF4 seems to be important for the survival of breast cancer cells, and this probably explains the oncogenic effect of KLF4 in these cells.

The authors conclude that the systemic targeting of KLF4 would not be desirable, as it would inhibit both the oncogenic and the tumour-suppressor functions of KLF4.

Nicola McCarthy

(2) References and links

ORIGINAL RESEARCH PAPER Rowland, B. D., Bernards, R. \& Peeper, D. S. The KLF4 tumour supressor is a transcriptional repressor of p53 that acts as a context-dependent oncogene. Nature Cell Biol. 23 Oct 2005 (doi 10.1038/ncb1314) 